\title{
SCIWORA NA POPULAÇÃO PEDIÁTRICA APÓS TRAUMATISMO CERVICAL
}

\author{
SCIWORA IN THE PAEDIATRIC POPULATION AFTER CERVICAL TRAUMA
}

SCIWORA EN LA POBLACIÓN PEDIÁTRICA DESPUÉS DE TRAUMATISMO CERVICAL

Vítor Duarte Gonçalves Vidinha1, António Pedro Cacho Rodrigues', Manuel Eduardo Cruz Ribeiro da Silva', Joana Moreira Fonseca

Barcelos Andrade'1, Nuno Silva Morais Neves², Rui Alexandre Peixoto Pinto ${ }^{3}$

\begin{abstract}
RESUMO
Objetivo: Avaliar a incidência e as características das lesões tipo SCIWORA nos indivíduos até os 16 anos, da área de um hospital central entre 1989 e 2009, após traumatismo cervical. Métodos: Estudo retrospectivo de consulta processual. Resultados: Nove (10,5\%) de 86 crianças apresentavam achados clínicos e radiológicos compatíveis com o diagnóstico de SCIWORA. A média de idades foi de 10,7 anos. A causa mais frequente foi o acidente de trânsito. Seis indivíduos eram classificáveis como Frankel D. Os restantes três casos eram Frankel C. Em três doentes a RMN mostrou imagem de lesão. Sete efetuaram metilprednisolona endovenosa e todos mantiveram imobilização com colar cervical até a primeira consulta de seguimento, às 2 semanas. Na alta, os seis doentes que apresentavam Frankel $D$ à entrada melhoraram para um grau E. Dos doentes com Frankel $\mathrm{C}$ à entrada, um melhorou até Frankel $\mathrm{D}$ e os restantes dois mantiveram-se inalterados em Frankel C. Conclusão: Em um hospital de referência traumatológica, SCIWORA representa cerca de 10\% das lesões cervicais pediátricas. Os défices neurológicos à entrada e a RMN têm valor prognóstico de recuperação. A corticoterapia em dose elevada não está formalmente indicada e não é consensual o tempo de utilização de imobilização ou a sua indicação em todos os SCIWORA.
\end{abstract}

Descritores: Pediatria; Traumatismos da medula espinhal; Vértebras cervicais; Espectroscopia de ressonância magnética.

\begin{abstract}
Objective: To assess the incidence and characteristics of SCIWORA in individuals under 16 years, from the area of a Level 1 Trauma Center between 1989 and 2009 after cervical trauma. Methods: Retrospective study of clinical charts consultation. Results: Nine (10.5\%) of 86 children had clinical features compatible with the radiological diagnosis of SCIWORA. The mean age was 10.7 years. The most frequent cause was traffic accident. Six individuals were classified as Frankel D. The remaining three cases were Frankel $\mathrm{C}$. In three patients the MRI showed an image of lesion. Seven had intravenous methylprednisolone done and all remained with cervical collar immobilization until the first follow-up visit at 2 weeks. At discharge six patients who had Frankel $D$ at entrance improved to a grade $E$. Of the patients with Frankel $C$ at entry, one improved to Frankel $D$ and the other two remained unchanged in Frankel C. Conclusion: In a level 1 Trauma Center SCIWORA represents nearly $10 \%$ of all spine injuries. Neurological deficits at entry and MRI have prognostic value for recovery. High dosage corticosteroids is not formally indicated and there is no consensus as to how long cenvical immobilization should be prescribed or its indication at all in SCIWORA patients.
\end{abstract}

Keywords: Pediatrics; Spinal cord injuries; Cervical vertebrae; Magnetic resonance spectroscopy.

\section{RESUMEN}

Objetivo: Evaluar la incidencia y las características de las lesiones tipo SCIWORA en los individuos de hasta 16 años de edad, del área de un hospital central, entre 1989 y 2009, después de traumatismo cervical. Métodos: Estudio retrospectivo de consulta procesal. Resultados: Nueve (10,5\%) de 86 niños presentaban hallazgos clínicos y radiológicos compatibles con el diagnóstico de SC/WORA. El promedio de edades fue 10,7 años. La causa más frecuente fue el accidente de tránsito. Seis individuos eran clasificables como Frankel D. Los restantes tres casos eran Frankel C. En tres pacientes, la RMN mostró imagen de lesión. Siete recibieron metilprednisolona endovenosa y todos mantuvieron inmovilización con collar cervical hasta la primera consulta de seguimiento, después de 2 semanas. En la alta, los seis pacientes, que presentaban Frankel D a la entrada, mejoraron para un grado E. De los pacientes con Frankel C a la entrada, uno mejoró hasta Frankel D y los otros dos se mantuvieron, sin alteraciones, en Frankel C. Conclusión: En un hospital de referencia traumatológica, SCIWORA representa cerca de $10 \%$ de las lesiones cervicales pediátricas. Los déficits neurológicos a la entrada y a RMN tienen valor de pronóstico de recuperación. La corticoterapia en dosis alta no está indicada formalmente y no es consensual el tiempo de utilización de inmovilización o su indicación en todos los SCIWORA.

Descriptores: Pediatría; Traumatismos de la médula espinal; Vértebras cenvicales; Espectroscopia de resonancia magnética.

1. Interno de Formação Específica de Ortopedia eTraumatologia no Centro Hospitalar de São João - EPE - Faculdade de Medicina da Universidade do Porto - Portugal.

2. Assistente Hospitalar do Serviço de Ortopedia eTraumatologia do Centro Hospitalar de São João - EPE - Faculdade de Medicina da Universidade do Porto - Portugal.

3. Director do Serviço de Ortopedia eTraumatologia do Centro Hospitalar de São João - EPE - Faculdade de Medicina da Universidade do Porto - Portugal.

Trabalho realizado no Grupo da Coluna - Serviço de Ortopedia - Centro Hospitalar de São João - EPE - Faculdade de Medicina da Universidade do Porto - Portugal.

Correspondência: Serviço de Ortopedia - Centro Hospitalar de São João - EPE - Alameda Prof. Hernâni Monteiro 4200 - Porto - Portugal.

E-mail: vitorgoncalvesvidinha@gmail.com

Recebido em 16/12/2010, aceito em 15/03/2011. 


\section{INTRODUÇÃO}

A patologia cervical traumática é pouco comum em idade pediátrica. Viccellio et al. ${ }^{1}$, em 2001, obtiveram uma incidência de $1 \%$, num estudo envolvendo mais de 3000 crianças. No entanto, nalgumas séries representa 60 a $80 \%$ de todas as lesões por traumatismo da coluna vertebral na criança, contrastando com os valores de 30 a $40 \%$ da idade adulta ${ }^{2-4}$. Diferenças fisiológicas e anatómicas deste segmento corporal estão na base da discrepância de valores nas duas faixas etárias.

A referência na literatura a lesão medular sem lesão vertebral na criança data do início dos anos vinte ${ }^{5}$. Em 1982 Pang e Wildberger ${ }^{6}$ introduzem o termo SCIWORA, que define um sub-grupo de patologias traumáticas da coluna vertebral com mielopatia associada objectiva, sem tradução radiográfica de lesão (fractura ou luxação) em crianças. A prevalência nesse estudo foi de $67 \%$. A incidência desta patologia varia muito nos diferentes estudos efectuados desde então. Ruge et al. ${ }^{4}$, verificaram uma incidência de 21,3\% num estudo retrospectivo de 1988, envolvendo 47 crianças.

Posteriormente a ocorrência desta lesão foi alargada à idade adulta onde ocorre com menor frequência e assume características próprias $^{7}$. Chen et al. ${ }^{8}$ mencionam cinco casos de compressão da medula após traumatismo na idade adulta, num artigo de 1997 e Kothari et al. ${ }^{9}$ publicam uma série de quatro casos em 2009.

No presente trabalho os autores propõe-se caracterizar clínica e epidemiologicamente as lesões da medula espinhal sem alterações radiográficas, na população pediátrica assistida no Serviço de Urgência de um hospital central, entre 1989 e 2009.

\section{MATERIAL E MÉTODOS}

Foram revistos retrospectivamente os registos clínicos de todos os pacientes com idade até aos 16 anos, inclusive, admitidos no Serviço de Urgência entre 1 de Janeiro de 1989 e 31 de Dezembro de 2009, com o diagnóstico de lesão traumática neurológica ou da coluna vertebral cervical. O diagnóstico de SCIWORA foi feito na presença de lesão neurológica traumática sem evidência radiográfica de fractura ou luxação vertebral.

As lesões neurológicas foram classificadas pela escala de Frankel.

\section{RESULTADOS}

Num total de 86 casos de crianças com idade até aos dezasseis anos, com diagnóstico de lesão da coluna cervical, nove (10,5\%) apresentavam achados clínicos (mielopatia) e exames radiológicos (sem alterações radiográficas) compatíveis com o diagnóstico de SCIWORA (Tabela 1). Destas, três eram do sexo masculino.
A média de idades foi de 10,7 anos, variando entre os nove meses e dezasseis anos. A causa havia sido um acidente de viação em cinco dos casos, sendo que os restantes resultaram de acidentes desportivos (mergulho - um; artes marciais - um; não especificados - dois). Seis indivíduos eram classificáveis como Frankel D. Os restantes três casos eram Frankel $\mathrm{C}$. Foram efectuados exames de imagiologia: radiografias cervicais simples de face e perfil $(n=8)$, radiografias dinâmicas $(n=1)$; tomografia computorizada cervical $(n=2)$ e ressonância magnética nuclear cervical $(n=8)$. Em três doentes a RMN mostrou imagem de lesão (Figuras 1 e 2).

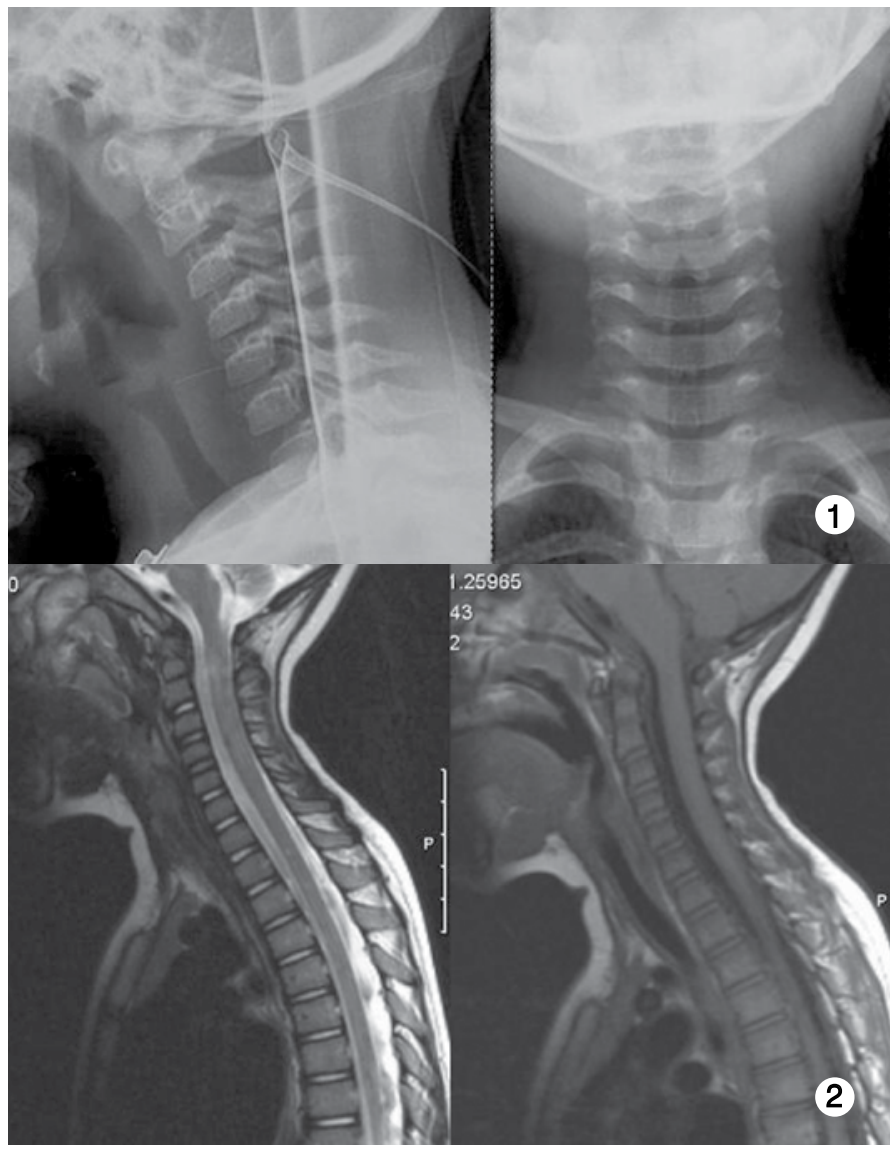

Figuras 1 e 2. Doente do sexo feminino, 2 anos, Frankel $C$ à entrada. RX não mostrou alterações. RMN evidenciando contusão medular extensa. Sem recuperação neurológica.

Tabela 1. Resumo dos doentes com diagnóstico de SCIWORA.

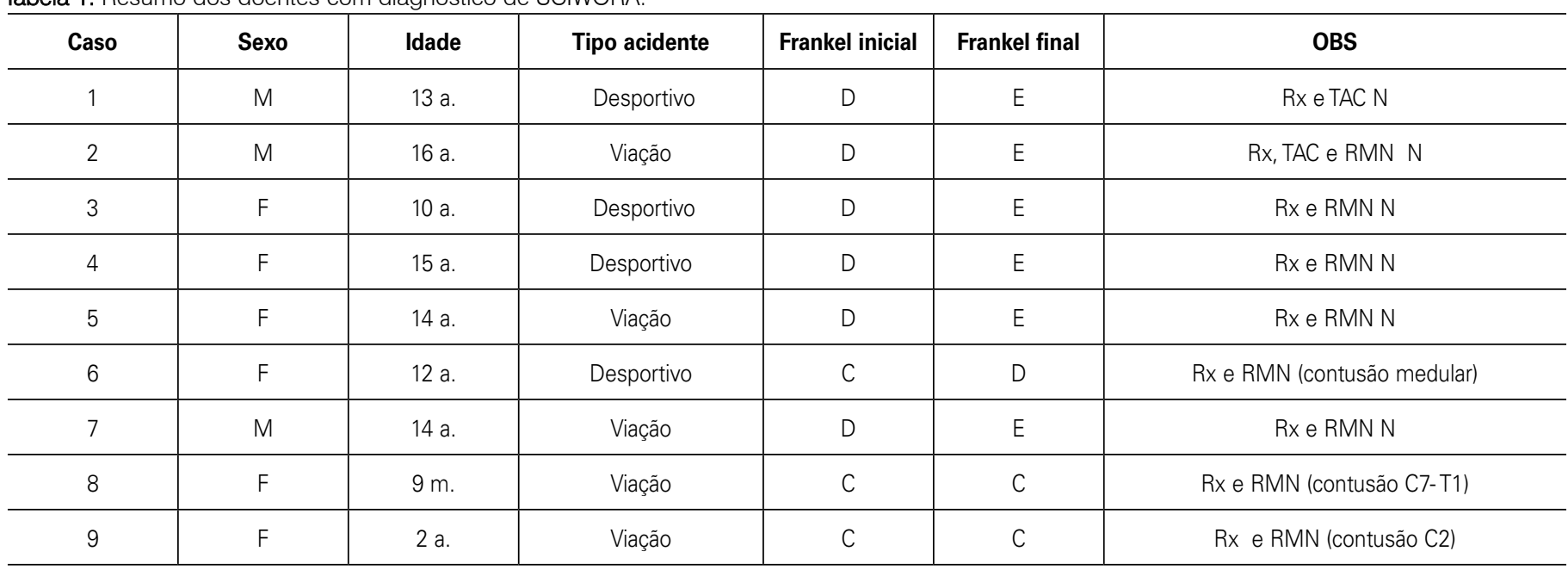


Durante o internamento, sete efectuaram metilprednisolona endovenosa e todos mantiveram imobilização com colar cervical até à primeira consulta de seguimento, às 2 semanas após a alta.

À data da alta todos os seis doentes que apresentavam Frankel $D$ à entrada melhoraram para um grau $E$, o que corresponde a um estado neurológico intacto. Um dos doentes com Frankel $\mathrm{C}$ à entrada melhorou até Frankel $\mathrm{D}$, e os restantes dois mantiveram-se inalterados em Frankel C.

\section{DISCUSSÃO}

Tem vindo a verificar-se um interesse crescente por esta patologia desde a sua descrição em 1982, e muitos centros têm efectuado estudos com base nas suas séries de doentes. No entanto, os dados estatísticos obtidos são díspares o que dificulta o estabelecimento de valores epidemiológicos para esta entidade. Nalguns centros, o diagnóstico de SCIWORA é aplicado de forma menos restrita comparativamente a outros. Bosch et al. ${ }^{10}$ ao reverem uma base de dados de doentes diagnosticados com SCIWORA, no seu hospital, notaram que a amostra era composta por um grupo muito heterogéneo de indivíduos, muitos dos quais não tinham na documentação escrita défice medular objectivo descrito e outros apresentavam um exame neurológico normal poucas horas depois da admissão. Na nossa série obtivemos uma incidência de 10,5\%, valor que se situa dentro do intervalo inferior a $20 \%$ referido na maioria dos estudos publicados ${ }^{11}$. É provável que diferentes critérios diagnósticos estejam na base dos diferentes valores estatísticos verificados na literatura.

Neste estudo apenas uma das crianças tinham idade inferior a nove anos (casos 8 e 9). Até esta idade as características morfológicas e fisiológicas que determinam uma maleabilidade inerente à coluna cervical que a distingue da do adulto são mais notórias ${ }^{12}$ e, por isso, teoricamente, crianças com idade inferior a este valor apresentariam maior susceptibilidade para a ocorrência de SCIWORA. Hadley et al. ${ }^{13}$ num estudo comparativo entre dois grupos, um constituído por sujeitos com idades compreendidas entre os zero e os nove anos e o outro com idades dos dez aos dezasseis anos, constataram uma maior incidência desta patologia no grupo dos mais novos. Osenbach e Menezes ${ }^{14}$ e Hamilton e Myles ${ }^{15}$ relataram resultados semelhantes. Por outro lado, num estudo que utilizou a base de dados $\mathrm{Nexus}^{16}$, constituída por 34069 indivíduos, havia 818 com lesão da coluna cervical, 27 dos quais com SCIWORA. Todos estes indivíduos eram adultos, e das 3000 crianças incluídas, nenhuma tinha critérios de SCIWORA.

Especulamos que a maior incidência em indivíduos com idade superior a nove anos na nossa série possa ter várias causas: 1) o desconhecimento da patologia por parte dos médicos que prestam cuidados a estes doentes; 2) o registo incompleto da informação no momento da colheita da anamnese; 3) a existência de erros na codificação de patologias, aquando do arquivamento do processo.

Perante um traumatismo, a instabilidade da coluna cervical do nascimento aos 9 anos protege a estrutura óssea de fractura ou luxação, mas propicia uma lesão da medula espinhal mais grave do que numa criança mais velha ${ }^{12}$. Num estudo de Pang e Polla$\mathrm{ck}^{17}$, envolvendo 55 crianças, apenas uma das 22 crianças com lesão neurológica profunda tinha mais de oito anos. A maioria das crianças com lesão severa não recupera ${ }^{6}$ e nesta série os doentes com pior estado neurológico à entrada (casos 6, 8 e 9), mantiveram défices neurológicos permanentes, tendo-se verificado a recuperação total nos restantes. Na nossa casuística não há evidência da recorrência de SCIWORA, embora esteja descrita ${ }^{17}$.

É possível que esta patologia esteja a ser subestimada nas faixas etárias mais velhas dado que a gravidade que assume em termos de estado neurológico é menor comparativamente aos indivíduos mais novos.

Quando inicialmente descrita, a SCIWORA era um diagnóstico de exclusão, caracterizado pela ausência de lesão detectável nos exames de imagem (radiografias de face, perfil, dinâmicas e tomografia computorizada) perante a clínica de lesão neurológica. 0 advento da ressonância magnética permitiu constatar de facto, a existência de lesão tecidular ${ }^{18}$. Para além disso, este exame tem correlação prognóstica significativa com a recuperação neurológica ${ }^{19}$.

Uma das críticas que se podem fazer à nossa investigação tem que ver com o facto de apenas dois doentes terem feito tomografia computorizada. É verdade que na descrição inicial se postula que este exame deve ser normal para excluir lesão óssea. No entanto, é consensual que um exame radiográfico bem executado identifica a grande maioria das lesões ósseas ${ }^{20}$. Para além disso, Stassen et al. ${ }^{21}$ mostraram resultados de concordância total entre a RMN e a TC a avaliar lesão óssea cervical (todos os 31 pacientes com RMN negativa tinham TC negativa para lesão e dos oito pacientes com TC positiva, todos apresentavam RMN positiva). A nosso ver, é pouco provável que estes doentes pudessem ter uma lesão óssea não visualizada nos RX e na RMN efectuada, e num contexto clínico que nos faz suspeitar de lesão neurológica sem lesão óssea associada parece-nos aceitável partir para a RMN, evitando a radiação inerente à realização de tomografia computorizada.

A literatura científica é contraditória no que respeita ao papel da ressonância magnética no diagnóstico de SCIWORA. Pang ${ }^{12}$, no seu artigo de revisão de 2004, chama a atenção para o facto da RMN ter evidenciado dano em virtualmente todos os tecidos de suporte não ósseos (a base para a anteriormente postulada instabilidade oculta $)^{6}$ e mostrado cinco classes de achados na medula espinhal pós-SCIWORA (secção completa, hemorragia major, hemorragia minor, edema e normal). Dos oito doentes que no nosso estudo efectuaram RMN, três apresentavam alteração do sinal medular. Estes mesmos doentes não recuperaram a função neurológica até à normalidade. É conhecido o papel da RMN no prognóstico dos doentes com SCIWORA. Davis et al. ${ }^{22}$ descreveram sete casos de crianças com SCIWORA e verificaram uma correlação entre contusão da medula e défices permanentes, bem como a associação entre uma RMN normal e um resultado neurológico final normal. Grabb e Pang ${ }^{23}$ descrevem achados semelhantes para sete crianças com radiografias normais que foram estudadas com ressonância magnética, e concluíram que achados normais associam-se a recuperação neurológica completa, contrastando com achados de hemorragia major ou descontinuidade da medula, associados a lesões completas permanentes.

Sete dos doentes efectuaram terapêutica com corticóides. Não há registo ou suspeita de complicações atribuíveis à administração deste fármaco nesta série. Porém, literatura recente salienta que altas doses de metilprednisolona não demonstraram benefício, mas pelo contrário, morbilidade significativa ${ }^{24}$. Para além disso, os estudos mais citados da administração de esteróides em lesões medulares não incluíram doentes com menos de 14 anos $^{25}$. Finalmente, a utilização de corticoterapia na lesão medular aguda não é um tratamento baseado na evidência, de acordo com uma revisão recente da literatura existente ${ }^{26}$. Face a estes dados, actualmente defendemos a não administração de corticoterapia a doentes pediátricos com SCIWORA.

A literatura científica existente não é consensual no que respeita ao tempo de imobilização dos doentes com diagnóstico de SCIWORA. Pang e Pollack ${ }^{17}$, num artigo de 1989, advogam a imobilização com colar cervical até às 12 semanas, baseado num caso de SCIWORA recorrente às 10 semanas, relativo a um doente que já havia recuperado totalmente a função sensitivo-motora do primei- 
ro episódio, e que manteve défices permanentes. Outros autores elaboraram os seus algoritmos de actuação baseados neste caso. No entanto, nesse mesmo artigo duas do total de oito crianças que tiveram um SCIWORA recorrente usavam colar, ou seja, a imobilização não preveniu a recorrência. Bosch et al. ${ }^{10}$ fazem referência a este facto, e defendem que a imobilização deve ser mantida até que a cervicalgia tenha desaparecido, o exame neurológico tenha normalizado e os exames auxiliares de diagnóstico apropriados (RX dinâmicos ou RMN) sejam negativos para instabilidade. Pang ${ }^{12}$ posteriormente reviu o seu algoritmo de actuação defendendo a imobilização durante três meses em doentes com alterações visualizáveis na RMN ou com défices neurológicos persistentes, e a imobilização com reavaliação às duas semanas nos doentes com estado neurológico normalizado e sem imagens sugestivas de lesão na RMN. A questão fundamental é se existe de facto uma instabilidade intersegmentar associada ao SCIWORA, se esta instabilidade é detectável pelos exames auxiliares de diagnóstico existentes e se o colar cervical anula essa instabilidade, prevenindo a recorrência de SCIWORA, o que ainda não está presentemente estabelecido na literatura. Na nossa instituição defendemos a imobilização com colar cervical até aos 15 a 30 dias, altura em que deve ser retirado se assintomático, ou efectuadas as radiografias dinâmicas, sob vigilância médica, se sintomático.

\section{CONCLUSÃO}

Num hospital de referência traumatológica SCIWORA representa cerca de $10 \%$ das lesões cervicais pediátricas.

Défices neurológicos francos à entrada e alterações medulares na RMN indicam um pior prognóstico de recuperação.

Face à evidência actual, a corticoterapia em dose elevada não está formalmente indicada.

Não é consensual o tempo de utilização de imobilização ou a indicação inequívoca em todos os casos de SCIWORA.

\section{REFERÊNCIAS}

1. Viccellio $P$, Simon $H$, Pressman BD, Shah MN, Mower WR, Hoffman JR, et al. A prospective multicenter study of cervical spine injury in children. Pediatrics.2001;108(2):E20.

2. Reynolds R. Pediatric spinal injury. Curr Opin Pediatr. 2000;12(1):67-71.

3. Avellino AM, Mann FA, Grady MS, Chapman JR, Ellenbogen RG, Alden TD, et al. The misdiagnosis of acute cervical spine injuries and fractures in infants and children: the 12-year experience of a level I pediatric and adult trauma center. Childs Nerv Syst. 2005;21(2):122-7.

4. Ruge JR, Sinson GP, McLone DG, Cerullo LJ. Pediatric spinal injury: the very young. J Neurosurg. 1988;68(1):25-30.

5. Crothers B. Injury of the spinal cord in Breech extraction as an important cause of fetal death and of paraplegia in childhood. Am J Med Sci. 1923;165:94-110.

6. Pang D, Wilberger JE Jr. Spinal cord injury without radiographic abnormalities in children. J Neurosurg. 1982;57(1):114-29.

7. Kasimatis GB, Panagiotopoulos E, Megas P, Matzaroglou C, Gliatis J,Tyllianakis M, et al. The adult spinal cord injury without radiographic abnormalities syndrome: magnetic resonance imaging and clinical findings in adults with spinal cord injuries having normal radiographs and computed tomography studies. J Trauma. 2008;65(1):86-93.

8. Chen TY, Lee ST, Lui TN, Wong CW, Yeh YS, Tzaan WC, Hung SY. Efficacy of surgical treatment in traumatic central cord syndrome. Surg Neurol. 1997:48(5):435-40.

9. Kothari P, Freeman B, Grevitt M, Kerslake R. Injury to the spinal cord without radiological abnormality (SCIWORA) in adults. J Bone Joint Surg Br. 2000;82(7):1034-7.

10. Bosch PP, Vogt MT, WardWT. Pediatric spinal cord injury without radiographic abnormality (SCIWORA): the absence of occult instability and lack of indication for bracing. Spine (Phila Pa 1976). 2002;27(24):2788-800

11. Grubenhoff JA, Brent A. Case report: Brown-Séquard syndrome resulting from a ski injury in a 7-year-old male. Curr Opin Pediatr. 2008;20(3):341-4

12. Pang D. Spinal cord injury without radiographic abnormality in children, 2 decades later. Neurosurgery. 2004;55(6):1325-42.

13. Hadley MN, Zabramski JM, Browner CM, Rekate H, Sonntag VK. Pediatric spinal trauma. Review of 122 cases of spinal cord and vertebral column injuries. J Neurosurg. 1988;68(1):18-24.

14. Osenbach RK, Menezes AH. Pediatric spinal cord and vertebral column injury. Neurosurgery. 1992;30(3):385-90.
15. Hamilton MG, Myles ST. Pediatric spinal injury: review of 174 hospital admissions. J Neurosurg. 1992;77(5):700-4.

16. Hendey GW, Wolfson AB, Mower WR, Hoffman JR; National Emergency X-Radiography Utilization Study Group. Spinal cord injury without radiographic abnormality: results of the National Emergency X-Radiography Utilization Study in blunt cervical trauma. J Trauma. 2002:53(1):1-4.

17. Pang D, Pollack IF. Spinal cord injury without radiographic abnormality in children--the SCIWORA syndrome. J Trauma. 1989;29(5):654-64.

18. Matsumura A, Meguro K, Tsurushima H, Kikuchi Y, Wada M, Nakata Y. Magnetic resonance imaging of spinal cord injury without radiologic abnormality. Surg Neurol. 1990:33(4):281-3

19. Liao CC, Lui TN, Chen LR, Chuang CC, Huang YC. Spinal cord injury without radiological abnormality in preschool-aged children: correlation of magnetic resonance imaging findings with neurological outcomes. J Neurosurg. 2005;103(1 Suppl):17-23.

20. Mower WR, Hoffman JR, Pollack CV Jr, Zucker MI, Browne BJ, Wolfson AB et al. Use of plain radiography to screen for cervical spine injuries. Ann Emerg Med. 2001;38(1):1-7.

21. Stassen NA, Williams VA, Gestring ML, Cheng JD, Bankey PE. Magnetic resonance imaging in combination with helical computed tomography provides a safe and efficient method of cervical spine clearance in the obtunded trauma patient. J Trauma. 2006;60(1):171-7.

22. Davis PC, Reisner A, Hudgins PA, Davis WE, O'Brien MS. Spinal injuries in children: role of MR. AJNR Am J Neuroradiol. 1993;14(3):607-17.

23. Grabb PA, Pang D. Magnetic resonance imaging in the evaluation of spinal cord injury without radiographic abnormality in children. Neurosurgery. 1994;35(3):406-14.

24. Macias MY, Maiman MJ. Critical care of acute spinal cord injuries. In: Jallo J, AR Vaccaro, editors. Neurotrauma and critical care of the spine. New York: Thieme Medical Publishers; 2009. p.171-84

25. Bracken MB, Shepard MJ, Collins WF, Holford TR, Young W, Baskin DS, et al. A randomized, controlled trial of methylprednisolone or naloxone in the treatment of acute spinal-cord injury. Results of the Second National Acute Spinal Cord Injury Study. N Engl J Med. 1990:322(20):1405-11.

26. Miranda P, Gomez P, Alday R, Kaen A, Ramos A. Brown-Sequard syndrome after blunt cervical spine trauma: clinical and radiological correlations. Eur Spine J. 2007;16(8):1165-70 